\title{
MicroRNA-3690 promotes cell proliferation and cell cycle progression by altering DKK3 expression in human thyroid cancer
}

\author{
FEI SHEN ${ }^{1,2^{*}}$, XIAO-XIONG GAN ${ }^{1,2^{*}}$, XING-YAN DENG ${ }^{1,2^{*}}$, JIAN-HUA FENG $^{1,2}$, \\ WEN-SONG CAI ${ }^{2}$, LIANG SHEN ${ }^{2}$, HUAN-QING XIAO ${ }^{2}$ and BO XU ${ }^{1,2}$ \\ ${ }^{1}$ Department of Thyroid Surgery, Guangzhou First People's Hospital, Guangzhou Medical University; \\ ${ }^{2}$ Department of Thyroid Surgery, Guangzhou First People's Hospital, School of Medicine, South \\ China University of Technology, Guangzhou, Guangdong 510180, P.R. China
}

Received April 13, 2019; Accepted July 20, 2020

DOI: $10.3892 / 01.2020 .12086$

\begin{abstract}
An increasing amount of evidence has demonstrated the importance of microRNAs (miRNAs/miRs) in the tumorigenesis of malignant types of cancer, and data retrieved from The Cancer Genome Atlas database revealed that miR-3690 was upregulated in thyroid cancer (TC). The present study focused on the biological function and mechanism of miR-3690 in TC, demonstrating that miR-3690 expression was significantly elevated in TC cells and clinical tissues. Functional studies indicated that miR-3690 acted as an oncogene in TC by promoting cell proliferation, colony formation and cell cycle progression in association with the increased expression of cyclin E and c-myc. Mechanistically, prediction software indicated that Dickkopf-related protein 3 (DKK3) was a target of miR-3690, which was confirmed by the results of luciferase reporter assays and western blotting. DKK3 silencing abrogated the functions of miR-3690-in on TC cell proliferation. Collectively, the findings of the present study demonstrated that miR-3690 promoted TC cell proliferation and indicated miR-3690 as a potential biomarker and therapeutic target for TC.
\end{abstract}

\section{Introduction}

Thyroid cancer (TC) is the most common endocrine malignancy and its incidence has increased rapidly in recent years worldwide (1). Due to the absence of obvious symptoms in the early stages of TC, $30 \%$ of patients are diagnosed at a

Correspondence to: Dr Bo Xu, Department of Thyroid Surgery, Guangzhou First People's Hospital, School of Medicine, South China University of Technology, 1 Panfu Road, Yuexiu, Guangzhou, Guangdong 510180, P.R. China

E-mail: gzsrmxu@yeah.net

${ }^{*}$ Contributed equally

Key words: microRNA-3690, human thyroid cancer, Dickkopf-related protein 3, cell proliferation, the cell cycle late stage with lymph node metastases or invasion of the surrounding tissues and organs (2). Furthermore, 10-20\% of patients exhibit distant metastases and recurrence, decreasing the 10-year survival rate to $40 \%$ (3-5). In addition, selected TC cases are not sensitive to radio-iodine treatment, and the subsequent curative effect is poor (6). Therefore, it is of great importance to understand the molecular mechanisms underlying TC in order to develop and improve current therapeutic approaches.

MicroRNAs (miRNAs) are a class of short endogenous non-coding molecules ( 22 nucleotides in length) that play key roles in oncogenesis and cancer progression by regulating various biological functions (7-9). Accumulating evidence has suggested that aberrant miRNA expression contributes to multiple physiological and pathological processes during TC (10-12). For example, Boufraqech et al (13) indicated that miR-30a was downregulated in TC, decreasing cellular invasion and migration by regulating lysyl oxidase. In addition, miR-338-3p was revealed to suppress cell proliferation, clonogenicity and metastasis in TC by targeting RAC- $\gamma$ serine/threonine-protein kinase (14). miR-215 was reported to inhibit TC cell proliferation, migration and invasion via the AKT/glycogen synthase kinase-3 $\beta /$ Snail signaling pathway by targeting brefeldin A-inhibited guanine nucleotide-exchange protein 1 (15). Furthermore, Guan et al (16) indicated that the downregulation of miR-218-2 promoted the invasion and progression of TC by regulating platelet-derived growth factor receptor $\alpha$ and 1-phosphatidylinositol 4,5-bisphosphate phosphodiesterase $\gamma-1$. The aim of the present study was to systematically investigate the precise role of miR-3690 in TC and elucidate the underlying mechanism, in order to determine the value of miR-3690 as a biomarker for TC.

\section{Materials and methods}

Clinical specimens and expression datasets. Clinical tissue samples were collected from 4 male and 4 female patients (mean age, $35 \pm 8$ years) who underwent total thyroidectomy (cancerous samples were collected from unilateral glandular lobe, and non-cancerous samples were collected from unilateral glandular lobe on the other side) and who were histopathologically diagnosed with TC between January 2016 and January 2017 at 
Guangzhou First People's Hospital (Guangzhou, China). The samples were immediately cryopreserved in liquid nitrogen and stored at $-80^{\circ} \mathrm{C}$ until further use. In order to investigate the expression of miR-3690 in association with TC, the miR-3690 expression data of 59 patients were downloaded from The Cancer Genome Atlas (TCGA; https://cancergenome.nih.gov/) database and subsequently analyzed.

Cell culture. The human thyroid follicular cell line Nthy-ori 3-1, and various human TC cell lines, including TPC-1, SW1736, FRO, K1 and 8505C, were purchased from the National Rodent Laboratory Animal Resource. The TC cell lines were authenticated by STR profiling, and were cultured in RPMI-1640 medium (Invitrogen; Thermo Fisher Scientific, Inc.) containing 10\% FBS (Thermo Fisher Scientific, Inc.), $100 \mathrm{U} / \mathrm{ml}$ penicillin and $0.1 \mathrm{mg} / \mathrm{ml}$ streptomycin. Nthy-ori 3-1 cells were cultured in F-12K medium (Genom Biotech Pvt., Ltd.) containing 10\% FBS, $100 \mathrm{U} / \mathrm{ml}$ penicillin and $0.1 \mathrm{mg} / \mathrm{ml}$ streptomycin, and all cells were cultured in a humidified atmosphere at $37^{\circ} \mathrm{C}$ $\left(5 \% \mathrm{Co}_{2}\right)$.

Plasmids, short interfering RNA (siRNA) and transfection. Synthetic miR-3690 (cat. no. miR10018119-1-5), miR-3690inhibitor (cat. no. miR20018119-1-5), miR-3690-mutant (mut, 5'-GCUUGG ACC CAGCGUAGACAA AG-3') and relative negative control miRNAs (vector: Cat.no.miR1N0000001-1-5, NC: Cat. no. miR2N0000001-1-5) were synthesized by Guangzhou RiboBio Co., Ltd. Cells transfections with $50 \mathrm{nM}$ miRNAs were performed using Lipofectamine $^{\circledR} 2000$ (Thermo Fisher Scientific, Inc.), according to the manufacturer's instructions.

Silencing DKK3 siRNAs (cat. no. HSH007526) were synthesized and purified by GeneCopoeia, Inc., and transfections were performed using Lipofectamine ${ }^{\circledR} 2000$ transfection reagent (Thermo Fisher Scientific, Inc.) according to the manufacturer's protocol. All cells were harvested for subsequent experimentation $48 \mathrm{~h}$ post-transfection.

$R N A$ extraction and reverse transcription-quantitative $P C R$ $(R T-q P C R)$ analysis. Total RNA from the human clinical tissues and cultured cells was extracted using TRIzol ${ }^{\circledR}$ reagent according to the manufacturer's recommendations (Thermo Fisher Scientific, Inc.). Reverse transcription and detection of mRNA (Cyclin E and c-myc) was performed using BlazeTaq $^{\mathrm{TM}}$ SYBR $^{\circledR}$ Green qPCR mix 2.0 (GeneCopoeia, Inc.). RT-qPCR was performed to detect miR-3690 expression using the All-in-One ${ }^{\mathrm{TM}}$ miRNA qRT-PCR Detection kit 2.0 (GeneCopoeia, Inc, Guangzhou). Thermocycling conditions were as follows: At $95^{\circ} \mathrm{C}$ for $30 \mathrm{sec}$, followed by 40 cycles of amplification at $95^{\circ} \mathrm{C}$ for $5 \mathrm{sec}$, at $59^{\circ} \mathrm{C}$ for $30 \mathrm{sec}$ and at $72^{\circ} \mathrm{C}$ for $30 \mathrm{sec}$. The following PCR primers were synthesized by GeneCopoeia, Inc.: miR-3690 (cat. no. HmiRQP1976), cyclin E (cat. no. HQP021819) and MYC (cat. no. HQP011597). U6 small nuclear RNA (cat. no. HmiRQP9001) and GAPDH (cat. no. HQP006940) were used as endogenous controls, and mRNA quantification was performed using the $2^{-\Delta \Delta C q}$ method (17).

MTT and anchorage-independent growth assays. In order to determine the cell proliferation rate, an MTT assay
(Sigma-Aldrich; Merck KGaA) was used according to the manufacturer's protocol. The reaction was stopped by $200 \mu \mathrm{l}$ DMSO; the relative optical density was determined at a wavelength of $490 \mathrm{~nm}$, and the results are expressed as the mean \pm standard deviation.

For the anchorage-independent growth assays, transfected $\mathrm{K} 1$ cells $\left(1 \times 10^{3}\right.$ cell per well) were seeded into $2 \mathrm{ml}$ complete medium with $0.3 \%$ soft agar (Invitrogen; Thermo Fisher Scientific, Inc.) and overlaid onto $2 \mathrm{ml}$ preset $1.5 \%$ agar in the same medium. The cells were cultured at $37^{\circ} \mathrm{C}$ with $5 \% \mathrm{CO}_{2}$ for 2 weeks, the colonies were then stained with $0.05 \%$ crystal violet for $10 \mathrm{~min}$ at room temperature, and colonies sized $>0.1 \mathrm{~mm}$ were counted under a microscope (Motic AE30 inverted fluorescence microscope; Microscope Systems Limited; magnification, $\mathrm{x} 40$ ).

Bioinformatics. The potential target genes of miR-3690 were predicted using Target Scan Human (version 7.1; http://www. targetscan.org/vert_71). Luciferase reporter assays. Based on the binding sites of DDK-3 and miR-3690, which were predicted using TargetScan, wild-type DDK-3 sequences were designed and cloned into the pGL3 luciferase reporter vector (Promega Corporation). Cells were then co-transfected with the vectors and miR-3690, miR-3690-in or miR-3690-mut, using Lipofectamine ${ }^{\circledR} 2000$ transfection reagent. Following a 48-h incubation, luciferase signals were measured using the Dual luciferase reporter gene assay kit (BioVision, Inc.) according to the manufacturer's protocol. Firefly luciferase activity was normalized to that of Renilla luciferase.

Western blotting. Transcfeted TC cells were lysed in protein RIPA buffer (cat. no. P0013; Beyotime Institute of Biotechnology) according to the manufacturer's protocol. The protein concentration was determined using a bicinchoninic acid protein assay. Proteins $(40 \mu \mathrm{g})$ were separated via $10 \%$ SDS-PAGE and subsequently transferred onto PVDF membranes (EMD Millipore). Membranes were blocked with $5 \%$ bovine serum albumin (BSA) for $1 \mathrm{~h}$ at room temperature, and incubated with primary antibodies against: DKK3 (1:1,000; cat. no. SAB2701251; Sigma-Aldrich; Merck KGaA), cyclin E (1:1,000; cat. no. 20808; Cell Signaling Technology, Inc.) and c-myc (1:1,000; cat. no. 18583; Cell Signaling Technology, Inc.) overnight at $4^{\circ} \mathrm{C}$, as previously described (17). To control sample loading, the membranes were stripped and re-probed with an anti- $\alpha$-tubulin antibody (1:500; cat. no. SAB5600206; Sigma-Aldrich; Merck KGaA). The membranes were then probed with a peroxidase-conjugated secondary antibody goat-anti-rabbit IgG (1:5,000; ZDR5306 or ZDR5307; Zhongshan Golden Bridge Biotechnology, Inc.), and the protein bands were quantified using Quantity One software version 4.6 (Bio-Rad Laboratories, Inc.).

Bromodeoxyuridine (BrdU) incorporation analysis. To assess cell proliferation, cells were cultured for $24 \mathrm{~h}$ following transfection. The medium was then supplemented with $10 \mathrm{hBrdU}$ and the cells were incubated for a further $6 \mathrm{~h}$. The labeled cells were thoroughly rinsed with PBS and incubated with an anti-BrdU antibody (1:500; cat no. 61273; Upstate Biotechnology, Inc.) for $1 \mathrm{~h}$ at $37^{\circ} \mathrm{C}$, according to the manufacturer's protocol. Images were captured using a laser scanning microscope (magnification, x100; Axioskop 2 plus; Carl Zeiss AG). 

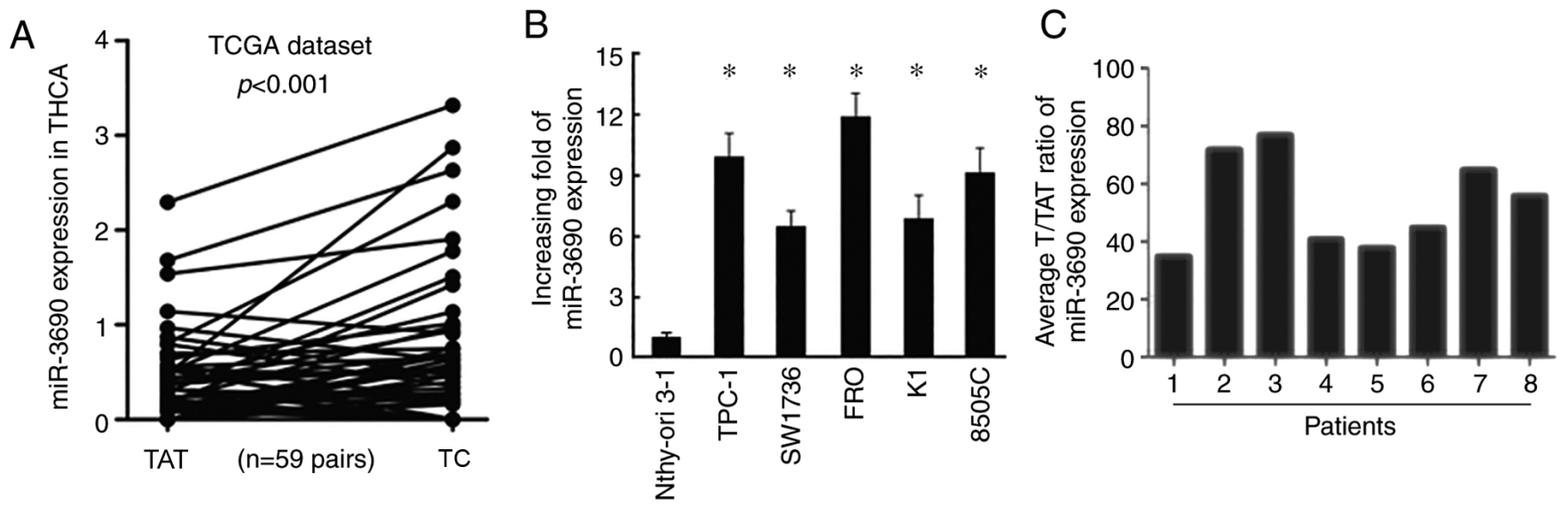

Figure 1. Expression of miR-3690 in human TC cell lines and clinical tissues. (A) The expression levels of miR-3690 in TC from TCGA dataset (n=59). (B) Reverse transcription PCR analysis of miR-3690 expression in primary Nthy-ori 3-1 (non-tumor follicular cell line) and human TC cell lines, including TPC-1, SW1736, FRO, K1 and 8505C. (C) Relative miR-3690 expression levels in 8 paired primary TC tissues (TC) and the tumor-adjacent normal tissues (TAT) from the same patient were detected by PCR analysis. Each bar represents the mean of three independent experiments. ${ }^{*}<0.05$ vs Nthy-ori $3-1$. miR, microRNA; TC, thyroid cancer; TCGA, The Cancer Genome Atlas; TAT, tumor-adjacent normal tissues.

Cellcycle distributionanalysis.Following miRNA-transfection for $48 \mathrm{~h}$, the cells were harvested, washed in ice-cold PBS, and fixed with pre-chilled $75 \%$ ethanol at $4^{\circ} \mathrm{C}$ overnight. The cells were stained using a propidium iodide/RNase staining buffer (BD Biosciences) at room temperature for $30 \mathrm{~min}$ in the dark, and the cell cycle distribution was analyzed using a FACSCalibur ${ }^{\mathrm{TM}}$ flow cytometer (BD Biosciences) according to the manufacturer's instructions.

Statistical analysis. Statistical analysis was performed using SPSS 17.0 (SPSS, Inc.). The data were analyzed using a paired Student's t-test for pair-wise comparisons or one-way analysis of variance followed by a post hoc Tukey test for multiple comparisons. $\mathrm{P}<0.05$ was considered to indicate a statistically significant difference.

\section{Results}

Upregulation of miR-3690 in TC clinical tissues and cell lines. Based on the expression data downloaded from TCGA, the expression level of miR-3690 was found to be higher in TC tissues compared with that in adjacent non-tumor tissues (Fig. 1A). Consistently, miR-3690 expression was also significantly increased in TC cell lines (TPC-1, SW1736, FRO, $\mathrm{K} 1$ and $8505 \mathrm{C}$ ) compared with that in human thyroid follicular cells (Nthy-ori 3-1; Fig. 1B). Furthermore, RT-qPCR analysis of samples from 8 patients with TC revealed significantly increased levels of miR-3690 in TC tissues (T) compared with tumor-adjacent normal tissues (TAT; Fig. 1C).

miR-3690 promotes TC cell proliferation, which is counteracted by miR-3690-in. To determine the effects of miR-3690 on cellular proliferation, TC cells were transfected with miR-3690 and assessed using RT-qPCR, MTT and anchorage-independent growth assays, in addition to BrdU labeling, immunofluorescence and cell cycle analysis. Following transfection of $\mathrm{K} 1$ cells, the expression level of miR-3690 was found to be significantly upregulated (Fig. 2A), and miR-3690 overexpression facilitated cellular proliferation
(Fig. 2B-D). Furthermore, miR-3690 overexpression significantly decreased the number of cells in the $G_{1} / G_{0}$ phase, but increased the proportion of $\mathrm{S}$ phase cells, compared with the control at $48 \mathrm{~h}$ post-transfection (Fig. 2E). In turn, miR-3690 expression was successfully inhibited by miR-3690-in transfection in K1 cells (Fig. 3A), which significantly suppressed proliferation and cell cycle progression compared with the control-transfected cells (Fig. 3B-E). Further to the effects of miR-3690 on TC cell proliferation, the results of the MTT and anchorage-independent growth assays revealed that the SW1736 cell line exhibited a lower miR-3690 expression level compared with the K1 line, and thus was selected for further experimentation. As presented in Fig. S1, the results reflected those obtained from K1 cells.

miR-3690 regulates DKK3 via the 3'-UTR and alters cell proliferation and the expression of cell cycle-associated genes. In order to identify the potential targets of miR-3690, a bioinformatics analysis was conducted using TargetScan, and revealed that DKK3 contains a putative binding site for miR-3690 (Fig. 4A). The effect of miR-3690-mut on TC cells was detected via RT-PCR analysis, the result showed that no effects were observed following transfection with miR-3690-mut compared with that in NC (Fig. 4B). To investigate the potential regulatory effect of miR-3690 on DKK3, protein expression levels were examined by western blot analysis in the present study. miR-3690 overexpression significantly decreased the expression levels of the DKK3 protein in $\mathrm{K} 1$ cells, while the levels were found to be upregulated following miR-3690-in transfection (Fig. 4C). Furthermore, luciferase reported assays indicated that miR-3690 significantly decreased the luciferase activity of the wild-type 3'-UTR of DKK3, whereas miR-3690-in transfection resulted in increased activity; no suppressive effects were observed following transfection with miR-3690-mut (Fig. 4D). These findings indicate that DKK3 is a direct target of miR-3690.

As miR-3690 promoted cell proliferation and cell cycle progression, regulatory genes associated with these processes were detected. RT-qPCR and western blot analysis revealed 
A

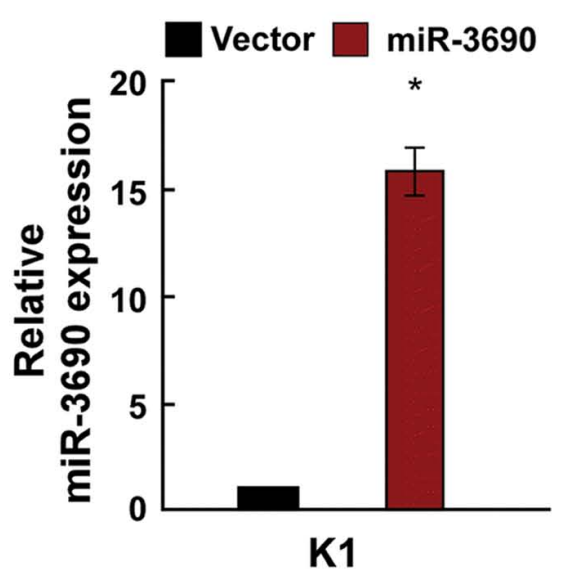

C
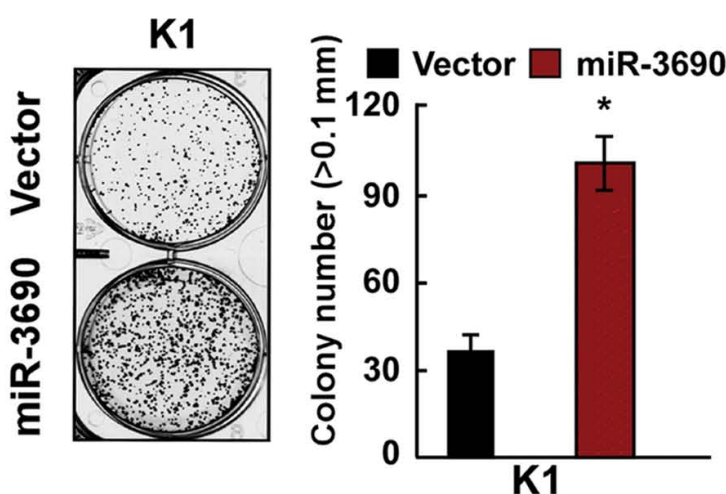

B

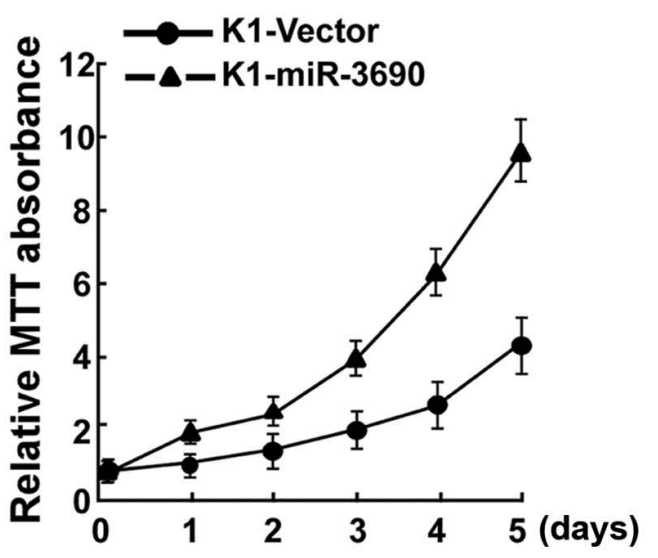

D

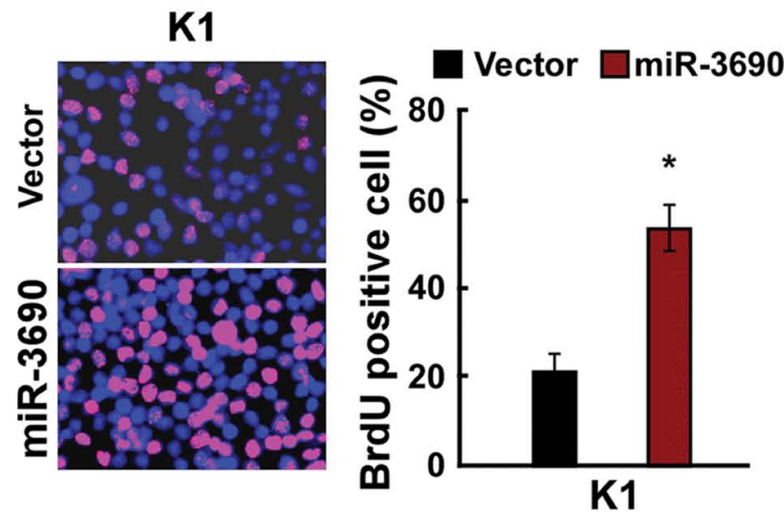

E
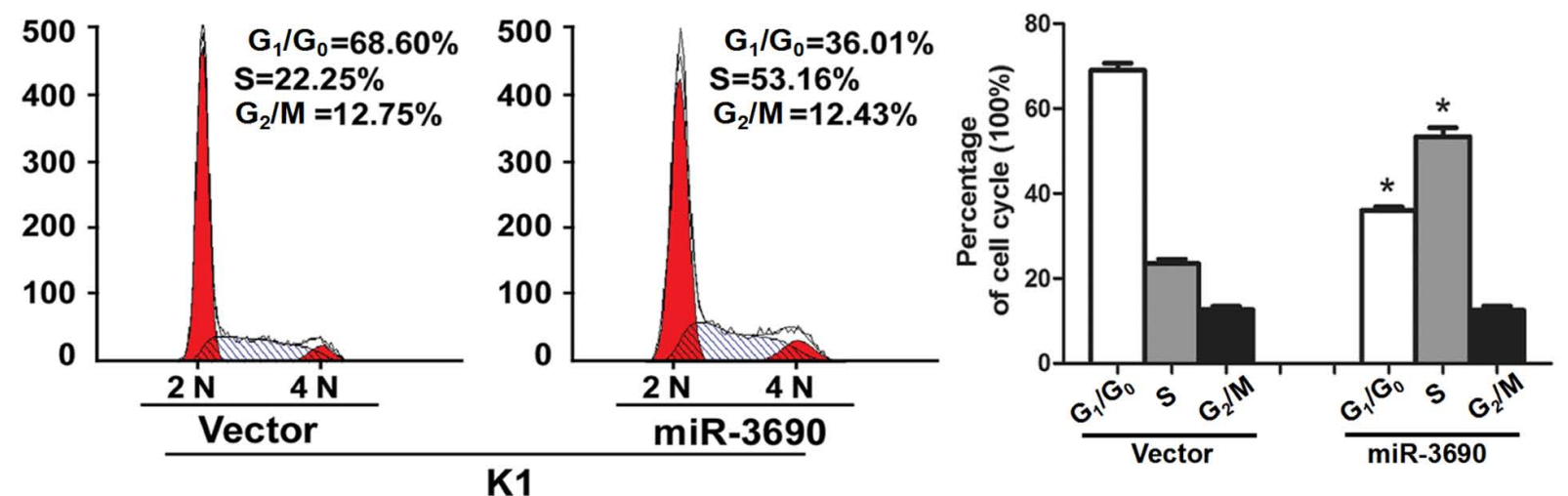

Figure 2. Upregulation of miR-3690 promoted TC cell proliferation. (A) Validation of miR-3690 expression levels after transfection by PCR analysis. (B) MTT assays revealed that overexpression of miR-3690 promoted proliferation of K1 cells. (C) Upregulation of miR-3690 promoted the anchorage-independent growth of K1 cells. Representative micrographs (left) and quantification of colonies that were $>0.1 \mathrm{~mm}$ (right). (D) Representative micrographs (left) and quantification (right) of the Brd incorporation assay in K1 cells. (E) Flow cytometric analysis of the indicated TC cell line K1 cells transfected with NC or miR-3690. Each bar represents the mean of three independent experiments. " $\mathrm{P}<0.05$ vs. vector group. TC, thyroid cancer; miR, microRNA; NC, negative control.

elevated mRNA and protein expression levels, respectively, of cyclin E and c-myc in miR-3690-transfected cells, which was consistent with the increased proportion of $\mathrm{S}$ phase and decreased proportion of $\mathrm{G}_{1} / \mathrm{G}_{0}$ phase cells (Fig. $4 \mathrm{E}$ and $\mathrm{F}$ ). By contrast, decreased expression of cyclin $\mathrm{E}$ and c-myc was detected following transfection with miR-3690-in, which was reflected by cell cycle arrest in the $\mathrm{G}_{1} / \mathrm{G}_{0}$ phase (Fig. $4 \mathrm{D}$ and $\mathrm{E}$ ).
DKK3 knockdown counteracts miR-3690-in-associated proliferative arrest. As DKK3 was confirmed to be a direct target of miR-3690, the effects of DKK3 downregulation on miR-3690-in-induced proliferative arrest were investigated. Following treatment with miR-3690-in, DKK3 was knocked down in K1 cells using specific siRNAs; knockdown efficiency was confirmed by western blotting (Fig. 5A). The 
A

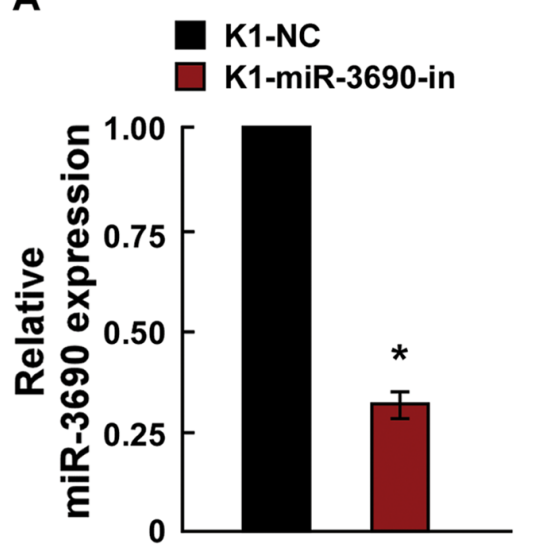

C

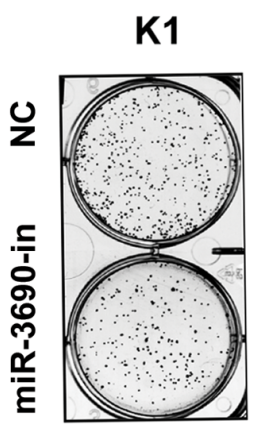

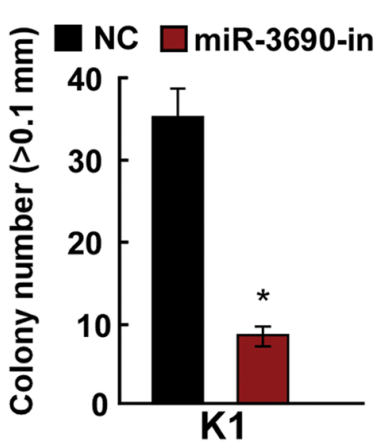

B

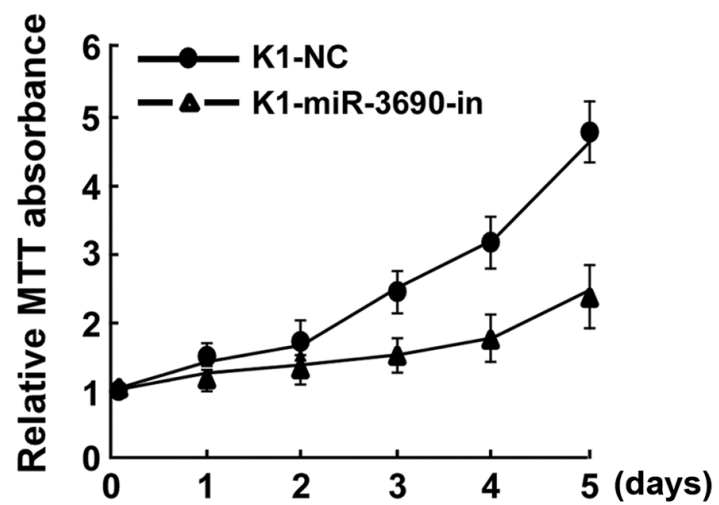

D

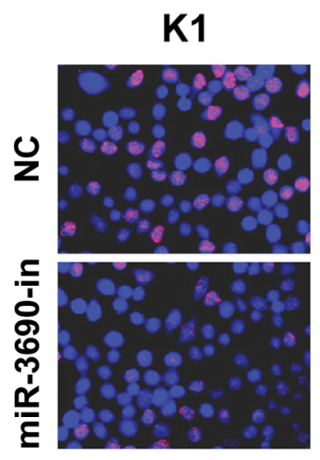

E
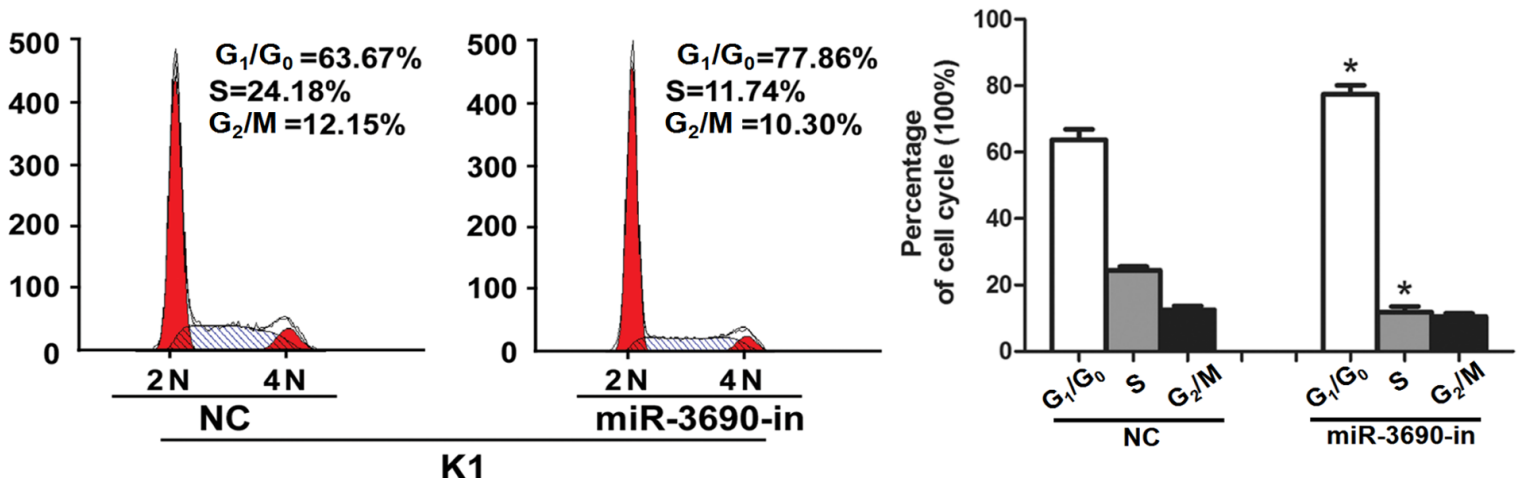

Figure 3. Inhibition of miR-3690 inhibited TC cell proliferation. (A) Validation of miR-3690 expression levels after transfection by PCR analysis. (B) MTT assays revealed that miR-3690-in suppressed growth of K1 cells. (C) Inhibition of miR-3690 inhibited the anchorage-independent growth of K1 cells Representative micrographs (left) and quantification of colonies that were $>0.1 \mathrm{~mm}$ (right). (D) Representative micrographs (left) and quantification (right) of the BrdU incorporation assay in K1 cells. (E) Flow cytometric analysis of the indicated TC cell line K1 cells transfected with NC or miR-3690-in. Each bar represents the mean of three independent experiments. "P<0.05 vs. NC group. miR, microRNA; TC, thyroid cancer; NC, negative control; miR-3690-in, microRNA-3690-inhibitor.

growth-suppressive effect of miR-3690-in was partially abrogated by DKK3-knockdown, as demonstrated by anchorage-independent growth (Fig. 5B), BrdU labeling and immunofluorescence assays (Fig. 5C). These findings indicate that DKK3 was involved in the miR-3690-in-induced suppression of cellular proliferation.

\section{Discussion}

Profiling studies have indicated that miRNAs play an essential role in TC development by regulating their target genes. miR-195 was found to suppress tumor growth and metastasis in TC by targeting $\mathrm{G}_{1} / \mathrm{S}$-specific cyclin $\mathrm{D} 1$ and fibroblast growth factor 2 (18); additionally, miR-577 was reported to inhibit the proliferation, migration and invasive capacity of TC cells by targeting sphingosine kinase 2 (19). The findings of Ye et al (20) indicated that fibronectin 1 was a direct target of miR-139, which inhibits TC progression. Furthermore, Sun et al (21) revealed that miR-144 suppressed TC cell proliferation through WW domain-containing transcription regulator protein 1 . Our previous study revealed that miR-639 promoted cell proliferation and cell cycle 
A
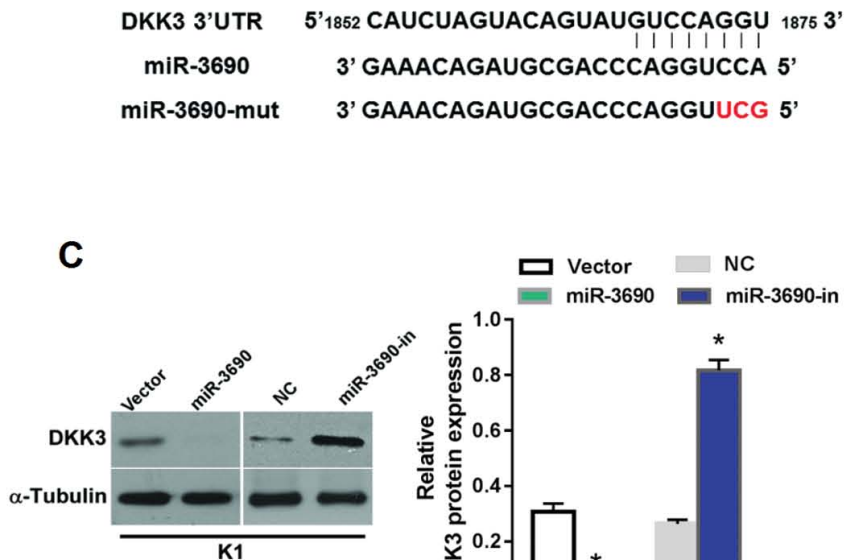

$\mathbf{E}$
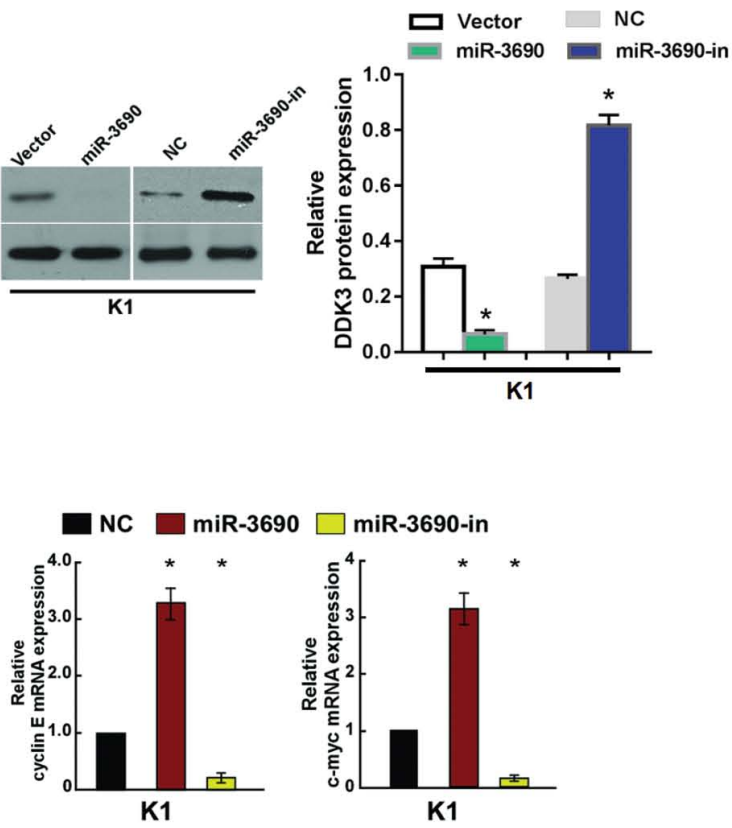

B

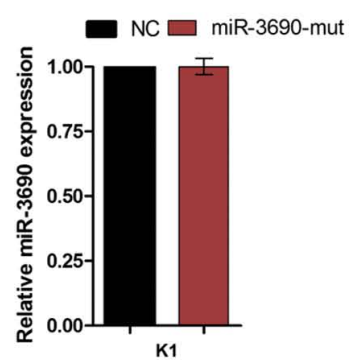

D

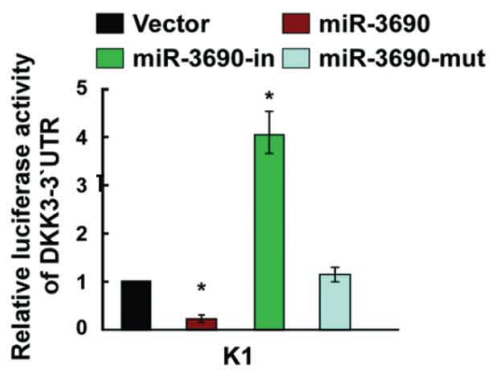

$\mathbf{F}$

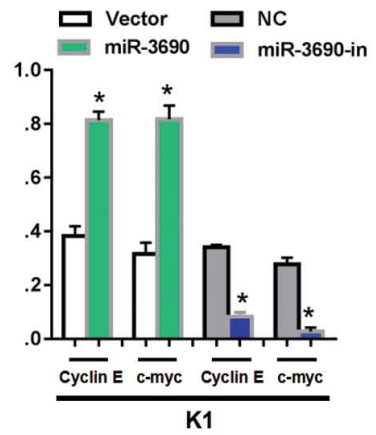

Figure 4. miR-3690 suppressed DKK3 expression by directly targeting its 3'-UTR and altered levels of proteins related to cell proliferation and cell cycle in K1 cells. (A) Predicted miR-3690 target sequence in the 3'-UTR of DKK3 (DKK3-3'-UTR) and positions of three mutated nucleotides (red) in the 3'-UTR of miR-3690 (miR-3690-mut). (B) Validation of miR-3690 expression levels after transfection with miR-3690-mut by PCR analysis. (C) Western blotting analysis of DKK3 expression in K1 cells transfected with miR-3690 or the miR-3690-in, $\alpha$-tubulin served as the loading control. (D) Luciferase reporter assay of the indicated cells transfected with the pGL3-DKK3-3'-UTR reporter and miR-3690 or miR-3690-in or miR-3690-mut. (E) Reverse transcription PCR analysis of expression of Cyclin E and c-myc in indicated K1 cells. (F) Western blotting analysis of expression of Cyclin E and c-myc protein in K1 cells. $\alpha$-tubulin served as the loading control. "P<0.05 vs. NC group or vector group. miR, microRNA; 3'-UTR, 3'-untranslated region; DKK3, Dickkopf-related protein 3; miR-3690-mut, microRNA-3690-mutant; miR-3690-in, microRNA-3690-inhibitor; NC, negative control.
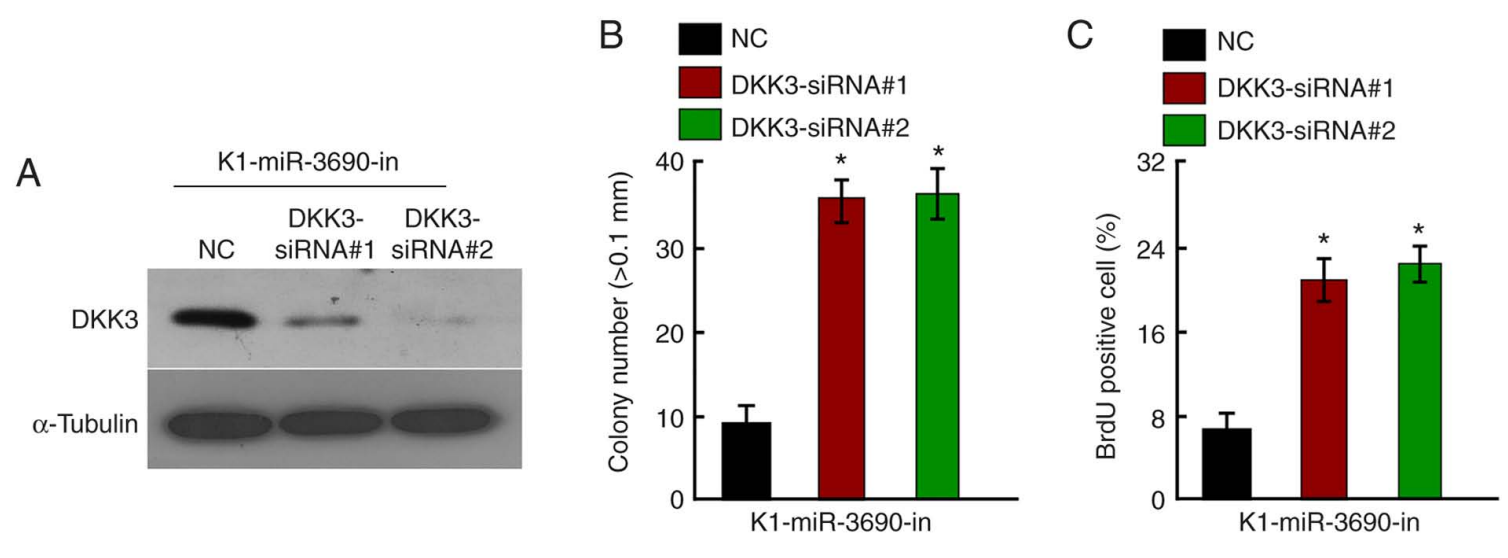

Figure 5. Downregulation of DKK3 counteracted the proliferation arrest by miR-3690-in. (A) Western blot analysis verified that silencing DKK3 effectively decreased the expression of DKK3 in miR-3690-in-transfected K1 cells. (B) miR-3690-in-transfected K1 cells after transfection with DKK3-siRNAs promoted the anchorage-independent growth. Representative quantification of colonies that were $>0.1 \mathrm{~mm}$. (C) Representative quantification of the BrdU incorporation assay. Each bar represents the mean of three independent experiments. "P<0.05. miR-3690-in, microRNA-3690-inhibitor; DKK3, Dickkopf-related protein 3; siRNA, small interfering-RNA; NC, negative control.

progression in TC by inhibiting cyclin-dependent kinase inhibitor 1 (22). Data from the present study provide evidence that miR-3690 expression is upregulated in TC tissues from patients and TC cell lines, and also suggest that miR-3690 
promotes cell proliferation and cell cycle progression by directly suppressing DKK3.

Human DKK3 is a member of the Dickkopf family, which perturbs the negative regulator Kremen and enhances Wnt signaling via low-density lipoprotein receptor-related protein 6 (23). Previous studies have demonstrated that DKK can act as a tumor promoter or suppressor in certain types of cancer, thereby affecting tumor development and progression. DDK3 has been reported to stimulate pancreatic ductal adenocarcinoma growth and metastasis, as well as resistance to chemotherapy, by activating NF- $\kappa \mathrm{B}$ (24). In addition, overexpression of DKK3 reportedly contributes to tumor cell proliferation, invasion, migration and survival in head and neck squamous cell carcinoma by inducing PI3K-Akt signaling (25). Furthermore, Xi et al (26) observed that DKK3 was downregulated in prostatic cancer, and that miR-95-3p contributed to the development of prostatic cancer by repressing DKK3, thus activating the Wnt/ $\beta$-catenin pathway. In gastric cancer, DKK3 was shown to attenuate CD133-induced NK-cell activation by inhibiting the Erk pathway and immunological synapse formation (27). DKK3 was also found to act as a biomarker and a therapeutic target by modulating Wnt signaling in several types of human cancer (28). However, the role of DKK3 in TC remains understudied. To the best of our knowledge, the present study was the first to demonstrate that DKK3 was a downstream target gene of miR-3690, and is considered to act as a tumor suppressor in TC. Furthermore, DKK3 downregulation counteracted miR-3690-in-associated proliferative arrest.

The present study revealed that miR-3690 promotes cellular proliferation by regulating proliferation- and cell cycle-associated genes, including cyclin E and c-myc. Cyclin $\mathrm{E}$ is a key checkpoint protein for G1-to-S phase transition in the cell cycle (29). C-myc is a known master regulator of the cell cycle and plays a critical role in carcinogenesis (30). In gastric carcinoma, downregulated DKK3 (REIC) expression was closely associated with aggressive phenotypes and poor prognosis (31). Ectopic DKK3 expression downregulated $\beta$-catenin, cyclin D2 and E expression levels, as well as regulated the cell cycle in gastric carcinoma (31). The results of the present study revealed that downregulation of miR-3690 resulted in cell cycle arrest at the $G_{1} / G_{0}$ phase in TC.

In conclusion, the present study demonstrated that miR-3690 was upregulated and promoted cancer cell proliferation by directly repressing DKK3. Downregulation of miR-3690 resulted in cell cycle arrest at the $\mathrm{G}_{1} / \mathrm{G}_{0}$ phase by inhibiting cyclin $\mathrm{E}$ and c-myc, which may affect the development of TC. These findings indicate that miR-3690 may be of value as a therapeutic target in TC.

\section{Acknowledgements}

Not applicable.

\section{Funding}

The present study was supported by Guangzhou Science and Technology Plan Project (grant no. 201805010003).

\section{Availability of data and materials}

The datasets generated or analyzed during the present study are included in this published article.

\section{Authors' contributions}

FS and BX designed the present study. FS, XG, XD and BX drafted the initial manuscript. FS, XG, XD and JF performed the experiments. FS, XG, XD, JF, WC, LS and HX analyzed the data. All authors read and approved the final manuscript.

\section{Ethics approval and consent to participate}

The present study was approved by the Ethics Committee of Guangzhou First People's Hospital (Guangzhou, China; approval no. 185-01), and written informed consent was provided by all patients.

\section{Patient consent for publication}

Not applicable.

\section{Competing interests}

The authors declare that they have no competing interests.

\section{References}

1. Chen W, Zheng R, Baade PD, Zhang S, Zeng H, Bray F, Jemal A, Yu XQ and He J: Cancer statistics in China, 2015. CA Cancer J Clin 66: 115-132, 2016.

2. Zhao H, Huang T and Li H: Risk factors for skip metastasis and lateral lymph node metastasis of papillary thyroid cancer. Surgery 166: 55-60, 2019.

3. Cabanillas ME, McFadden DG and Durante C: Thyroid cancer. Lancet 388: 2783-2795, 2016.

4. Lim H, Devesa SS, Sosa JA, Check D and Kitahara CM: Trends in thyroid cancer incidence and mortality in the United States, 1974-2013. JAMA 317: 1338-1348, 2017.

5. Kitahara CM and Sosa JA: The changing incidence of thyroid cancer. Nat Rev Endocrinol 12: 646-653, 2016.

6. Wagner M, Khoury H, Bennetts L, Willet J, Lister J, Berto P, Ehreth J, Badia X, Grimaldi-Bensouda L and Goetghebeur M: Appraising the value of lenvatinib for radio-iodine refractory differentiated thyroid cancer (Rr-Dtc): A multi-country study applying holistic multicriteria decision analysis (Mcda). Value Health 18: PA477-PA478, 2015.

7. Pei ZJ, Zhang ZG, Hu AX, Yang F and Gai Y: miR-122-5p inhibits tumor cell proliferation and induces apoptosis by targeting MYC in gastric cancer cells. Pharmazie 72: 344-347, 2017.

8. Xu FF, Xie WF, Zha GQ, Chen HW and Deng L: MiR-520f promotes cell aggressiveness by regulating fibroblast growth factor 16 in hepatocellular carcinoma. Oncotarget 8: 109546-109558, 2017.

9. Liang WL, Cao J, Xu B, Yang P, Shen F, Sun Z, Li WL, Wang Q and Liu F: miR-892a regulated PPP2R2A expression and promoted cell proliferation of human colorectal cancer cells. Biomed Pharmacother 72: 119-124, 2015.

10. Tsai MM, Wang CS, Tsai CY, Huang CG, Lee KF, Huang HW, Lin YH, Chi HC, Kuo LM, Lu PH and Lin KH: Circulating microRNA-196a/b are novel biomarkers associated with metastatic gastric cancer. Eur J Cancer 64: 137-148, 2016.

11. Gong Y, Wu W, Zou X, Liu F, Wei T and Zhu J: MiR-26a inhibits thyroid cancer cell proliferation by targeting ARPP19. Am J Cancer Res 8: 1030-1039, 2018.

12. Sun J, Shi R, Zhao S, Li X, Lu S, Bu H, Ma X and Su C: E2F8, a direct target of miR-144, promotes papillary thyroid cancer progression via regulating cell cycle. J Exp Clin Cancer Res 36: 40, 2017. 
13. Boufraqech M, Nilubol N, Zhang L, Gara SK, Sadowski SM, Mehta A, He M, Davis S, Dreiling J, Copland JA, et al: miR30a inhibits LOX expression and anaplastic thyroid cancer progression. Cancer Res 75: 367-377, 2015.

14. Sui GQ, Fei D, Guo F, Zhen X, Luo Q, Yin S and Wang H: MicroRNA-338-3p inhibits thyroid cancer progression through targeting AKT3. Am J Cancer Res 7: 1177-1187, 2017.

15. Han J, Zhang M, Nie C, Jia J, Wang F, Yu J, Bi W, Liu B, Sheng R, $\mathrm{He} \mathrm{G}$, et al: miR-215 suppresses papillary thyroid cancer proliferation, migration, and invasion through the AKT/GSK-3 $3 /$ Snail signaling by targeting ARFGEF1. Cell Death Dis 10: 195, 2019.

16. Guan H, Wei G, Wu J, Fang D, Liao Z, Xiao H, Li M and Li Y: Down-regulation of miR-218-2 and its host gene SLIT3 cooperate to promote invasion and progression of thyroid cancer J Clin Endocrinol Metab 98: E1334-E1344, 2013.

17. Livak KJ and Schmittgen TD: Analysis of relative gene expression data using real-time quantitative PCR and the 2(-Delta Delta C(T)) method. Methods 25: 402-408, 2001.

18. Yin Y, Hong S, Yu S, Huang Y, Chen S, Liu Y, Zhang Q, Li Y and Xiao H: MiR-195 inhibits tumor growth and metastasis in papillary thyroid carcinoma cell lines by targeting CCND1 and FGF2. Int J Endocrinol 2017: 6180425, 2017.

19. Xue KC, Hu DD, Zhao L, Li N and Shen HY: MiR-577 inhibits papillary thyroid carcinoma cell proliferation, migration and invasion by targeting SphK2. Eur Rev Med Pharmacol Sci 21: 3794-3800, 2017.

20. Ye Y, Zhuang J, Wang G, He S, Ni J and Xia W: MicroRNA-139 targets fibronectin 1 to inhibit papillary thyroid carcinoma progression. Oncol Lett 14: 7799-7806, 2017.

21. Sun W, Lan X, Wang Z, Dong W, He L, Zhang T, Zhang P and Zhang H: MicroRNA-144 inhibits proliferation by targeting WW domain-containing transcription regulator protein 1 in papillary thyroid cancer. Oncol Lett 15: 1007-1013, 2018

22. Lei ST, Shen F, Chen JW, Feng JH, Cai WS, Shen L, Hu ZW and Xu B: MiR-639 promoted cell proliferation and cell cycle in human thyroid cancer by suppressing CDKN1A expression. Biomed Pharmacother 84: 1834-1840, 2016.

23. Ferrari N, Ranftl R, Chicherova I, Slaven ND, Moeendarbary E, Farrugia AJ, Lam M, Semiannikova M, Westergaard MCW, Tchou J, et al: Dickkopf-3 links HSF1 and YAP/TAZ signalling to control aggressive behaviours in cancer-associated fibroblasts. Nat Commun 10: 130, 2019.
24. Zhou L, Husted H, Moore T, Lu M, Deng D, Liu Y, Ramachandran V, Arumugam T, Niehrs C, Wang H, et al: Suppression of stromal-derived Dickkopf-3 (DKK3) inhibits tumor progression and prolongs survival in pancreatic ductal adenocarcinoma. Sci Transl Med 10: eaat3487, 2018.

25. Katase N, Nishimatsu SI, Yamauchi A, Yamamura M, Terada K, Itadani M, Okada N, Hassan NMM, Nagatsuka H, Ikeda T, et al: DKK3 overexpression increases the malignant properties of head and neck squamous cell carcinoma cells. Oncol Res 26: 45-58, 2018.

26. Xi M, Cheng L, Hua W, Zhou YL, Gao QL, Yang JX and Qi SY: MicroRNA-95-3p promoted the development of prostatic cancer via regulating DKK3 and activating Wnt/ $\beta$-catenin pathway. Eur Rev Med Pharmacol Sci 23: 1002-1011, 2019.

27. Xia P and Xu XY: DKK3 attenuates the cytotoxic effect of natural killer cells on CD133 ${ }^{+}$gastric cancer cells. Mol Carcinog 56: 1712-1721, 2017.

28. Hamzehzadeh L, Caraglia M, Atkin SL and Sahebkar A: Dickkopf homolog 3 (DKK3): A candidate for detection and treatment of cancers? J Cell Physiol 233: 4595-4605, 2018.

29. Jing L, Gong M, Lu X, Jiang Y, Li H and Cheng W: LINC01127 promotes the development of ovarian tumors by regulating the cell cycle. Am J Transl Res 11: 406-417, 2019.

30. Elliott B, Millena AC, Matyunina L, Zhang M, Zou J, Wang G, Zhang Q, Bowen N, Eaton V, Webb G, et al: Essential role of JunD in cell proliferation is mediated via MYC signaling in prostate cancer cells. Cancer Lett 448: 155-167, 2019.

31. Xu XY, Xia P, Yu M, Nie XC, Yang X, Xing YN,Liu YP, Takano Y and Zheng HC: The roles of REIC gene and its encoding product in gastric carcinoma. Cell Cycle 11: 1414-1431, 2012.

This work is licensed under a Creative Commons Attribution-NonCommercial-NoDerivatives 4.0 International (CC BY-NC-ND 4.0) License. 